\title{
A Note on Implicit and Explicit Mann Iterative Processes for Asymptotically $\phi$-Strongly Pseudocontractive Mappings
}

\author{
Luigi Muglia' ${ }^{1}$ and Yonghong Yao ${ }^{2}$ \\ ${ }^{1}$ Dipartimento di Matematica, Universitá della Calabria, 87036 Arcavacata di Rende, Italy \\ ${ }^{2}$ Department of Mathematics, Tianjin Polytechnic University, Tianjin 300387, China
}

Correspondence should be addressed to Luigi Muglia, muglia@mat.unical.it

Received 20 January 2012; Accepted 15 February 2012

Academic Editor: Giuseppe Marino

Copyright (C) 2012 L. Muglia and Y. Yao. This is an open access article distributed under the Creative Commons Attribution License, which permits unrestricted use, distribution, and reproduction in any medium, provided the original work is properly cited.

In this paper we prove the equivalence and the strong convergence of an explicit Mann iterative process and a modified implicit iterative process for asymptotically $\phi$-strongly pseudocontractive mappings in a uniformly smooth Banach space.

\section{Introduction}

Let $X$ be a Banach space and $X^{*}$ the dual space of $X$. Let $J$ denote the normalized duality mapping form $X$ into $2^{X^{*}}$ given by $J(x)=\left\{f \in X^{*}:\langle x, f\rangle=\|x\|^{2}=\|f\|^{2}\right\}$ for all $x \in X$, where $\langle\cdot, \cdot\rangle$ denotes the generalized duality pairing.

In 1972, Goebel and Kirk [1] introduced the class of asymptotically nonexpansive mappings as follows.

Definition 1.1. Let $K$ be a subset of a Banach space $X$. A mapping $T: K \rightarrow K$ is said asymptotically nonexpansive if, for each $x, y \in K$,

$$
\left\|T^{n} x-T^{n} y\right\| \leq k_{n}\|x-y\|,
$$

where $\left\{k_{n}\right\}_{n} \subset[1, \infty)$ is a sequence of real numbers converging to 1 .

Their scope was to extend the well-known Browder's fixed point theorem [2] to this class of mappings. 
This class is really more general than the class of nonexpansive mappings (see [1]). In 1991, Schu [3] introduced the class of asymptotically pseudocontractive mappings.

Definition 1.2 (see [3]). Let $X$ be a normed space, $K \subset X$, and $\left\{k_{n}\right\}_{n} \subset[1, \infty)$. A mapping $T: K \rightarrow K$ is said to be asymptotically pseudocontractive with the sequence $\left\{k_{n}\right\}_{n}$ if and only if $\lim _{n \rightarrow \infty} k_{n}=1$ and, for all $n \in \mathbb{N}$ and all $x, y \in K$, there exists $j(x-y) \in J(x-y)$ such that

$$
\left\langle T^{n} x-T^{n} y, j(x-y)\right\rangle \leq k_{n}\|x-y\|^{2},
$$

where $J$ is the normalized duality mapping.

Obviously every asymptotically nonexpansive mapping is asymptotically pseudocontractive but the converse is not valid: it is well known that $T:[0,1] \rightarrow[0,1]$ defined by $T x=\left(1-x^{2 / 3}\right)^{3 / 2}$ is not Lipschitz but asymptotically pseudocontractive [4].

In [3], Schu proved the following.

Theorem 1.3 (see [3]). Let $H$ be a Hilbert space and $A \subset H$ closed and convex; $L>0 ; T: A \rightarrow A$ completely continuous, uniformly L-Lipschitzian and asymptotically pseudocontractive with sequence $\left\{k_{n}\right\}_{n} \in[1, \infty) ; q_{n}:=2 k_{n}-1$ for all $n \in \mathbb{N} ; \sum_{n}\left(q_{n}^{2}-1\right)<\infty ;\left\{\alpha_{n}\right\}_{n},\left\{\beta_{n}\right\}_{n} \in[0,1] ; \epsilon \leq \alpha_{n} \leq \beta_{n} \leq b$ for all $n \in \mathbb{N}$, some $\epsilon>0$ and some $b \in\left(0, L^{-2}\left[\sqrt{1+L^{2}}-1\right]\right) ; x_{1} \in A ;$ for all $n \in \mathbb{N}$ define

$$
\begin{gathered}
z_{n}:=\beta_{n} T^{n}\left(x_{n}\right)+\left(1-\beta_{n}\right) x_{n}, \\
x_{n+1}:=\alpha_{n} T^{n}\left(z_{n}\right)+\left(1-\alpha_{n}\right) x_{n} .
\end{gathered}
$$

Then, $\left\{x_{n}\right\}_{n}$ converges strongly to some fixed point of $T$.

From 1991 to 2009, no fixed point theorem for asymptotically pseudocontractive mappings had been proved. First Zhou, in [5], completed this lack in the setting of Hilbert spaces proving (1) a fixed-point theorem for an asymptotically pseudocontractive mapping that is also uniformly L-Lipschitzian and uniformly asymptotically regular; (2) that the set of fixed points of $T$ is closed and convex; (3) the strong convergence of a CQ-iterative method. The literature on asymptotical-type mappings is wide (see e.g., [6-11]).

In 1974, Deimling [12], studying the zeros of accretive operators, introduced the class of $\varphi$-strongly accretive operators.

Definition 1.4. An operator $A$ defined on a subset $K$ of a Banach space $X$ is called $\varphi$-strongly accretive if

$$
\langle A x-A y, j(x-y)\rangle \geq \varphi(\|x-y\|)\|x-y\|
$$

where $\varphi: \mathbb{R}^{+} \rightarrow \mathbb{R}^{+}$is a strictly increasing function such that $\varphi(0)=0$ and $j(x-y) \in J(x-y)$.

Note that, in the special case in which $\varphi(t)=k t, k \in(0,1)$, we obtain a strongly accretive operator.

Since an operator $A$ is a strongly accretive operators if and only if $(I-A)$ is a strongly pseudocontractive mappings (i.e., $\left.\langle(I-A) x-(I-A) y, j(x-y)\rangle \leq k\|x-y\|^{2}, k<1\right)$, taking in 
account Definition 1.4, it is natural to study the class of $\varphi$-pseudocontractive mappings, that is, the maps such that

$$
\langle T x-T y, j(x-y)\rangle \leq\|x-y\|^{2}-\varphi(\|x-y\|)\|x-y\|
$$

where $\varphi: \mathbb{R}^{+} \rightarrow \mathbb{R}^{+}$is a strictly increasing function such that $\varphi(0)=0$. Of course the set of fixed points for this mappings contains, at most, only one point.

Here our attention is on the class of the asymptotically $\phi$-strongly pseudocontractions define as follows.

Definition 1.5. If $X$ is a Banach space and $K$ is a subset of $X$, a mapping $T: K \rightarrow K$ is said asymptotically $\phi$-strongly pseudocontraction if

$$
\left\langle T^{n} x-T^{n} y, j(x-y)\right\rangle \leq k_{n}\|x-y\|^{2}-\phi(\|x-y\|),
$$

where $j(x-y) \in J(x-y),\left\{k_{n}\right\}_{n} \subset[1, \infty)$ is converging to one and $\phi:[0, \infty) \rightarrow[0, \infty)$ is strictly increasing and such that $\phi(0)=0$.

One can note that if $T$ has fixed points then it is unique. In fact if $x, z$ are fixed points for $T$, then, for every $n \in \mathbb{N}$,

$$
\|x-z\|^{2}=\left\langle T^{n} x-T^{n} z, j(x-z)\right\rangle \leq k_{n}\|x-y\|^{2}-\phi(\|x-y\|),
$$

so, passing $n$ to $+\infty$, it results

$$
\|x-z\|^{2} \leq\|x-z\|^{2}-\phi(\|x-y\|) \Longrightarrow-\phi(\|x-y\|) \geq 0 .
$$

Since $\phi:[0, \infty) \rightarrow[0, \infty)$ is strictly increasing and $\phi(0)=0$, then $x=z$.

We now give two examples.

Example 1.6. The mapping $T x=x /(x+1)$, where $x \in[0,1]$, is asymptotically $\phi$-strongly pseudocontraction with $k_{n}=1$, for all $n \in \mathbb{N}$ and $\phi(t)=t^{3} /(1+t)$. However, $T$ is not strongly pseudocontractive, see [13].

Example 1.7. The mapping $T x=x /(1+\alpha x)$, where $x \in[0,1]$ and $\alpha$ is closing to zero, is asymptotically $\phi$-strongly pseudocontraction with $k_{n}=1+1 / \alpha n$, for all $n \in \mathbb{N}$ and $\phi(x)=x^{3} /(1+x)$. However, $T$ is not strongly pseudocontractive and nor is the $\phi$-strongly pseudocontraction.

Proof. First we prove that $T$ is not strongly pseudocontractive. For arbitrary $k<1$, there exists $x, y \in[0,1]$, such that

$$
\frac{1}{(1+\alpha x)(1+\alpha y)}>k
$$


So we have

$$
\langle T x-T y, j(x-y)\rangle=\frac{1}{(1+\alpha x)(1+\alpha y)}(x-y)^{2}>k\|x-y\|^{2} .
$$

Next we prove that $T$ is not $\phi$-strongly pseudocontractive. Taking $y=0$, we have, for all $x \in[0,1]$,

$$
\begin{aligned}
& \langle T x-T y, j(x-y)\rangle=\frac{x^{2}}{(1+\alpha x)} \\
& \|x-y\|^{2}-\phi(\|x-y\|)=\frac{x^{2}}{1+x}
\end{aligned}
$$

Therefore, $T$ is not $\phi$-strongly pseudocontractive. Finally, we prove that $T$ is asymptotically $\phi$-strongly pseudocontraction.

For arbitrary $x, y \in[0,1]$, without loss of generality, let $x>y$. Then,

$$
\begin{aligned}
\left\langle T^{n} x-T^{n} y, j(x-y)\right\rangle & =\frac{(x-y)^{2}}{(1+\alpha n x)(1+\alpha n y)}, \\
k_{n}\|x-y\|^{2}-\phi(\|x-y\|) & =\left(1+\frac{1}{\alpha n}\right)(x-y)^{2}-\frac{(x-y)^{3}}{1+(x-y)} \\
& =\left[1+\frac{1}{\alpha n}-\frac{(x-y)}{1+(x-y)}\right](x-y)^{2} \\
& =\frac{1+1 / \alpha n+1 / \alpha n(x-y)}{1+(x-y)}(x-y)^{2} .
\end{aligned}
$$

We only need to prove that

$$
1+x-y \leq(1+\alpha n x)(1+\alpha n y)\left[1+\frac{1}{\alpha n}+\frac{1}{\alpha n}(x-y)\right]
$$

Using $x>y$, this is easy.

Let us consider the well-known Mann iterative process defined as follows: for any given $z_{0} \in X$, the sequence $\left\{z_{n}\right\}_{n}$ defined by

$$
z_{n+1}=\left(1-\alpha_{n}\right) z_{n}+\alpha_{n} T^{n} z_{n}, \quad n \geq 0 .
$$


We can also introduce a modified implicit iterative process as follows: suppose $T$ is continuous, $\left(w_{n}^{\prime}\right)_{n}$ is bounded, and let $z_{0}^{\prime}$ be an initial point. Thus, we define

$$
z_{n}^{\prime}=\left(1-\alpha_{n}-\gamma_{n}\right) z_{n-1}^{\prime}+\alpha_{n} T^{n} z_{n}^{\prime}+\gamma_{n} w_{n}^{\prime}, \quad n \geq 1
$$

where $\left\{\alpha_{n}\right\}_{n}$ is a real sequence in [0,1] satisfying $\alpha_{n} k_{n}<1$ for all $n \geq 1$.

The algorithm is well defined. In fact, if $T$ is a continuous asymptotically strongly $\phi$ pseudocontraction, for every fixed $n$, the mapping $S_{n}$ defined by $S_{n} x:=\left(1-\alpha_{n}-\gamma_{n}\right) z_{n-1}+$ $\alpha_{n} T^{n} x+\gamma_{n} w_{n}$ is such that

$$
\left\langle S_{n} x-S_{n} y, j(x-y)\right\rangle=\left\langle T^{n} x-T^{n} y, j(x-y)\right\rangle \leq \alpha_{n} k_{n}\|x-y\|^{2},
$$

that is, $S_{n}$ is a continuous strongly pseudocontraction, for every fixed $n$. Then (see Theorem 13.1 in [14]), there exists a unique fixed-point of $S_{n}$ for each $n$.

This modified implicit method is inspired to a wide literature.

In 1995, Liu [15] introduced the following modified Ishikawa method:

$$
\begin{gathered}
y_{n}=\left(1-\beta_{n}\right) x_{n}+\beta_{n} T^{n} x_{n}+v_{n}, \\
x_{n+1}=\left(1-\alpha_{n}\right) x_{n}+\alpha_{n} T^{n} y_{n}+u_{n}, \quad n \geq 0,
\end{gathered}
$$

and he called it Ischikawa iteration process with errors (obviously posing $\beta_{n}=0$, he obtains the Mann iteration process with errors). This new class of methods with errors was studied, among the others, also by Chang in [16] in 2001, Chang et. al [17] in 2006, Gu in [18], Huang in [19], and Huang and Bu in 2007 [20].

In 2001, Chidume and Osilike [21] proved the strong convergence of the iterative method

$$
\begin{aligned}
& y_{n}=a_{n} x_{n}+b_{n} S x_{n}+c_{n} u_{n}, \\
& x_{n+1}=a_{n}^{\prime} x_{n}+b_{n}^{\prime} S y_{n} c_{n}^{\prime} v_{n},
\end{aligned}
$$

where $a_{n}+b_{n}+c_{n}=a_{n}^{\prime}+b_{n}^{\prime}+c_{n}^{\prime}=1, S x=x-T x+f$ ( $T$ a $\phi$-strongly accretive operator $)$, and $f \in X$, to a solution of the equation $T x=f$. Note that Chidume and Osilike did not use term with errors to indicate their methods.

In 2003, Chidume and Zegeye [22] studied the following iterative method:

$$
x_{n+1}=\left(1-\alpha_{n}\right) x_{n}+\alpha_{n} T x_{n}-\alpha_{n} \theta_{n}\left(x_{n}-x_{1}\right),
$$

where $T$ is a Lipschitzian pseudocontractive map with fixed points. The authors proved the strong convergence of the method to a fixed point of $T$ under opportune hypotheses on the control sequences $\left(\theta_{n}\right)_{n},\left(\lambda_{n}\right)_{n}$.

If we pose $w_{n}=z_{0}$ in (1.14) and $\gamma_{n}=\alpha_{n} \theta_{n}$, the modified Mann iterative process coincides with the Chideme and Zegeye's iterative method.

In this paper, we prove the equivalence between the implicit and the explicit modified Mann iterative method which involves an asymptotically $\phi$-strongly pseudocontractive mapping. 


\section{Preliminaries}

Throughout this paper, we will assume that $X$ is a uniformly smooth Banach space. It is well known that, if $X$ is uniformly smooth, then the duality mapping $J$ is single-valued and is norm to norm uniformly continuous on any bounded subset of $X$. In the sequel, we shall denote the single-valued duality mapping by $j$.

For the sake of completeness, we recall some definitions and conclusions.

Definition 2.1. An $X$ is said to be a uniformly smooth Banach space if the smooth module of $X$,

$$
\rho_{X}(t)=\sup \left\{\frac{1}{2}(\|x-y\|+\|x+y\|)-1:\|x\| \leq 1,\|y\| \leq t\right\}
$$

satisfies $\lim _{t \rightarrow 0}\left(\rho_{X}(t) / t\right)=0$.

Lemma 2.2 (see [23]). Let $X$ be a Banach space, and let $J: X \rightarrow 2^{X^{*}}$ be the normalized duality mapping. Then, for any $x, y \in X$, we have

$$
\|x+y\|^{2} \leq\|x\|^{2}+2\langle y, j(x+y)\rangle, \quad \forall j(x+y) \in J(x+y) .
$$

Next lemma is a key for our proofs.

Lemma 2.3 (see [19]). Let $\phi:[0, \infty) \rightarrow[0, \infty)$ be a strictly increasing function with $\phi(0)=0$, and let $\left\{a_{n}\right\}_{n},\left\{b_{n}\right\}_{n},\left\{c_{n}\right\}_{n}$, and $\left\{e_{n}\right\}_{n}$ be nonnegative real sequences such that

$$
\lim _{n \rightarrow \infty} b_{n}=0, \quad c_{n}=o\left(b_{n}\right), \quad \sum_{n=1}^{\infty} b_{n}=\infty, \quad \lim _{n \rightarrow \infty} e_{n}=0 .
$$

Suppose that there exists an integer $N_{1}>0$ such that

$$
a_{n+1}^{2} \leq a_{n}^{2}-2 b_{n} \phi\left(\left|a_{n+1}-e_{n}\right|\right)+c_{n}, \quad \forall n \geq N_{1}
$$

then $\lim _{n \rightarrow \infty} a_{n}=0$.

Proof. The proof is the same as in [19] but changing, in (2.4), $\left(a_{n+1}-e_{n}\right)$ with $\left|a_{n+1}-e_{n}\right|$.

Lemma 2.4 (see [24]). Let $\left\{s_{n}\right\}_{n},\left\{c_{n}\right\}_{n} \subset \mathbb{R}_{+},\left\{a_{n}\right\}_{n} \subset(0,1)$, and $\left\{b_{n}\right\}_{n} \subset \mathbb{R}$ be sequences such that

$$
s_{n+1} \leq\left(1-a_{n}\right) s_{n}+b_{n}+c_{n}
$$

for all $n \geq 0$. Assume that $\sum_{n} c_{n}<\infty$. Then, the following results hold.

(1) If $b_{n} \leq \beta a_{n}$ (where $\beta \geq 0$ ), then $\left\{s_{n}\right\}_{n}$ is a bounded sequence.

(2) If we have $\sum_{n} a_{n}=\infty$ and $\lim \sup _{n}\left(b_{n} / a_{n}\right) \leq 0$, then $s_{n} \rightarrow 0$ as $n \rightarrow \infty$. 
Journal of Applied Mathematics

Remark 2.5. If, in Lemma 2.3, we choose $e_{n}=0$, for all $n, \phi(t)=k t^{2}(k<1)$, then the inequality (2.4) becomes

$$
\begin{aligned}
& a_{n+1}^{2} \leq a_{n}^{2}-2 b_{n} k a_{n+1}^{2}+c_{n} \Longrightarrow \\
& a_{n+1}^{2} \leq \frac{1}{1+2 b_{n} k} a_{n}^{2}+\frac{c_{n}}{1+2 b_{n} k} \leq\left(1-\frac{2 b_{n} k}{1+2 b_{n} k}\right) a_{n}^{2}+\frac{c_{n}}{1+2 b_{n} k},
\end{aligned}
$$

where $\alpha_{n}:=2 b_{n} k /\left(1+2 b_{n} k\right)$ and $\beta_{n}:=c_{n} /\left(1+2 b_{n} k\right)$. In the hypotheses of Lemma 2.3, $\alpha_{n} \rightarrow 0$ as $n \rightarrow \infty, \sum_{n} \alpha_{n}=\infty$ and $\lim \sup _{n}\left(\beta_{n} / \alpha_{n}\right)=0$. So we reobtain Lemma 2.4 in the case $c_{n}=0$.

\section{Main Results}

The ideas of the proofs of our main theorems take in account the papers of Chang and Chidume et al. [16, 21, 25].

Theorem 3.1. Let $X$ be a uniformly smooth Banach space, and let $T: X \rightarrow X$ be asymptotically $\phi$-strongly pseudocontractive mapping with fixed point $x^{*}$ and bounded range.

Let $\left\{v_{n}\right\}_{n}$ be the sequence defined by

$$
\begin{gathered}
v_{0} \in X, \\
v_{n+1}=\left(1-\alpha_{n}\right) v_{n}+\alpha_{n} T^{n} v_{n}, \quad n \geq 0,
\end{gathered}
$$

where $\left\{\alpha_{n}\right\}_{n} \subset[0,1]$ satisfies

(i) $\lim _{n \rightarrow \infty} \alpha_{n}=0$,

(ii) $\sum_{n=1}^{\infty} \alpha_{n}=\infty$.

Then, for any initial point $v_{0} \in X$, the sequence $\left\{v_{n}\right\}_{n}$ strongly converges to $x^{*}$.

Proof. By the boundedness of the range of $T$ and by Lemma 2.4, we have that $\left\{v_{n}\right\}_{n}$ is bounded.

By Lemma 2.2, we observe that

$$
\begin{aligned}
\left\|v_{n+1}-x^{*}\right\|^{2} \leq & \left(1-\alpha_{n}\right)^{2}\left\|v_{n}-x^{*}\right\|^{2}+2 \alpha_{n}\left\langle T^{n} v_{n}-x^{*}, j\left(v_{n+1}-x^{*}\right)\right\rangle \\
\leq & \left(1-\alpha_{n}\right)^{2}\left\|v_{n}-x^{*}\right\|^{2}+2 \alpha_{n}\left\langle T_{n} v_{n}-x^{*}, j\left(v_{n+1}-x^{*}\right)-j\left(v_{n}-x^{*}\right)\right\rangle \\
& +2 \alpha_{n}\left\langle T_{n} v_{n}-x^{*}, j\left(v_{n}-x^{*}\right)\right\rangle \\
\leq & \left(1-\alpha_{n}\right)^{2}\left\|v_{n}-x^{*}\right\|^{2}+2 \alpha_{n}\left\langle T_{n} v_{n}-x^{*}, j\left(v_{n+1}-x^{*}\right)-j\left(v_{n}-x^{*}\right)\right\rangle \\
& +2 \alpha_{n} k_{n}\left\|v_{n}-x^{*}\right\|^{2}-2 \alpha_{n} \phi\left(\left\|v_{n}-x^{*}\right\|\right) \\
= & \left(1+\alpha_{n}^{2}-2 \alpha_{n}\right)\left\|v_{n}-x^{*}\right\|^{2}+2 \alpha_{n}\left\langle T_{n} v_{n}-x^{*}, j\left(v_{n+1}-x^{*}\right)-j\left(v_{n}-x^{*}\right)\right\rangle \\
& +2 \alpha_{n} k_{n}\left\|v_{n}-x^{*}\right\|^{2}-2 \alpha_{n} \phi\left(\left\|v_{n}-x^{*}\right\|\right) \\
= & \left\|v_{n}-x^{*}\right\|^{2}+\left(\alpha_{n}^{2}-2 \alpha_{n}+2 \alpha_{n} k_{n}\right)\left\|v_{n}-x^{*}\right\|^{2}-2 \alpha_{n} \phi\left(\left\|v_{n}-x^{*}\right\|\right)+2 \alpha_{n} \mu_{n},
\end{aligned}
$$


where $\mu_{n}:=\left\langle T_{n} v_{n}-x^{*}, j\left(v_{n+1}-x^{*}\right)-j\left(v_{n}-x^{*}\right)\right\rangle$. Let

$$
M:=\max \left\{\sup _{n}\left\|v_{n}-x^{*}\right\|, \sup _{n}\left\|T^{n} v_{n}-x^{*}\right\|\right\} .
$$

We have

$$
\begin{aligned}
\left\|v_{n+1}-x^{*}\right\|^{2} & \leq\left\|v_{n}-x^{*}\right\|^{2}+\left(\alpha_{n}^{2}+2 \alpha_{n}\left(k_{n}-1\right)\right) M-2 \alpha_{n} \phi\left(\left\|v_{n}-x^{*}\right\|\right)+2 \alpha_{n} \mu_{n} \\
& =\left\|v_{n}-x^{*}\right\|^{2}-\alpha_{n} \phi\left(\left\|v_{n}-x^{*}\right\|\right)-\alpha_{n}\left[\phi\left(\left\|v_{n}-x^{*}\right\|\right)-2 \mu_{n}-\left(\alpha_{n}+2\left(k_{n}-1\right)\right) M\right],
\end{aligned}
$$

so we can observe the following.

(1) $\mu_{n} \rightarrow 0$ as $n \rightarrow \infty$. In fact from the inequality

$$
\left|\left\|v_{n+1}-x^{*}\right\|-\left\|v_{n}-x^{*}\right\|\right| \leq\left\|v_{n+1}-v_{n}\right\| \leq \alpha_{n} M \longrightarrow 0, \quad \text { as } n \longrightarrow \infty,
$$

and, since $j$ is norm to norm uniformly continuous, then

$$
j\left(\left\|v_{n+1}-x^{*}\right\|\right)-j\left(\left\|v_{n}-x^{*}\right\|\right) \longrightarrow 0, \quad \text { as } n \longrightarrow \infty .
$$

(2) $\inf _{n}\left(\left\|v_{n}-x^{*}\right\|\right)=0$. In fact, if we suppose that $\sigma:=\inf _{n}\left(\left\|v_{n}-x^{*}\right\|\right)>0$, by the monotonicity of $\phi$,

$$
\phi\left(\left\|v_{n}-x^{*}\right\|\right) \geq \phi(\sigma)>0 .
$$

Thus, by (1) and by the hypotheses on $\alpha_{n}$ and $k_{n}$, the value $-\alpha_{n}\left[\phi\left(\left\|v_{n}-x^{*}\right\|\right)-2 \mu_{n}-\right.$ $\left.\left(\alpha_{n}+2\left(k_{n}-1\right)\right) M\right]$ is definitively negative. In this case, we conclude that there exists $N>0$ such that, for every $n>N$,

$$
\left\|v_{n+1}-x^{*}\right\|^{2} \leq\left\|v_{n}-x^{*}\right\|^{2}-\alpha_{n} \phi\left(\left\|v_{n}-x^{*}\right\|\right) \leq\left\|v_{n}-x^{*}\right\|^{2}-\alpha_{n} \phi(\sigma)
$$

and so

$$
\alpha_{n} \phi(\sigma) \leq\left\|v_{n}-x^{*}\right\|^{2}-\left\|v_{n+1}-x^{*}\right\|^{2} \quad \forall n>N
$$

In the same manner, we obtain that

$$
\phi(\sigma) \sum_{i=N}^{m} \alpha_{i} \leq \sum_{i=N}^{m}\left[\left\|v_{i}-x^{*}\right\|^{2}-\left\|v_{i+1}-x^{*}\right\|^{2}\right]=\left\|v_{N}-x^{*}\right\|^{2}-\left\|v_{m}-x^{*}\right\|^{2} .
$$

By the hypothesis $\sum_{n} \alpha_{n}=\infty$, the previous is a contradiction and it follows that $\inf _{n}\left(\left\|v_{n}-x^{*}\right\|\right)=0$. 
Then, there exists a subsequence $\left\{v_{n_{k}}\right\}_{k}$ of $\left\{v_{n}\right\}_{n}$ that strongly converges to $x^{*}$. This implies that, for every $\epsilon>0$, there exists an index $n_{k(\epsilon)}$ such that, for all $j \geq n_{k(\epsilon)},\left\|v_{n_{j}}-x^{*}\right\|<\epsilon$.

Now, we will prove that the entire sequence $\left\{v_{n}\right\}_{n}$ converges to $x^{*}$. Since the sequences in (3.4) are null sequences but $\sum_{n} \alpha_{n}=\infty$, then, for every $\epsilon>0$, there exists an index $\bar{n}(\epsilon)$ such that, for all $n \geq \bar{n}(\epsilon)$, it results that

$$
\left|\alpha_{n}\right|<\frac{1}{2 M} \min \left\{\epsilon, \frac{\phi(\epsilon / 2)}{2}\right\}, \quad\left|k_{n}-1\right|<\frac{\phi(\epsilon / 2)}{8 M}, \quad\left|\mu_{n}\right|<\frac{\phi(\epsilon / 2)}{8} .
$$

So, fixing $\epsilon>0$, let $n^{*}>\max \left(n_{k(e)}, \bar{n}(\epsilon)\right)$ with $n^{*}=n_{j}$ for a certain $n_{j}$. We will prove, by induction, that $\left\|v_{n^{*}+i}-x^{*}\right\|<\epsilon$ for every $i \in \mathbb{N}$. Let $i=1$, if not, it results that $\left\|v_{n^{*}+1}-x^{*}\right\| \geq \epsilon$. Thus,

$$
\epsilon \leq\left\|v_{n^{*}+1}-x^{*}\right\| \leq\left\|v_{n^{*}}-x^{*}\right\|+\alpha_{n^{*}}<\left\|v_{n^{*}}-x^{*}\right\|+\frac{\epsilon}{2 M} M=\left\|v_{n^{*}}-x^{*}\right\|+\frac{\epsilon}{2}
$$

that is, $\left\|v_{n^{*}}-x^{*}\right\|>\epsilon / 2$. By the strict increasing of $\phi$, then $\phi\left(\left\|v_{n^{*}}-x^{*}\right\|\right)>\phi(\epsilon / 2)$. By (3.4), it results

$$
\left\|v_{n^{*}+1}-x^{*}\right\|^{2}<\epsilon^{2}-\alpha_{n^{*}} \phi\left(\left\|v_{n^{*}}-x^{*}\right\|\right)-\alpha_{n^{*}}\left[\phi\left(\left\|v_{n^{*}}-x^{*}\right\|\right)-2 \mu_{n^{*}}-\left(\alpha_{n^{*}}+2\left(k_{n^{*}}-1\right)\right) M\right] .
$$

We can note that

$$
2 \mu_{n^{*}}+\left(\alpha_{n^{*}}+2\left(k_{n^{*}}-1\right)\right) M \leq \frac{\phi(\epsilon / 2)}{4}+\left(\frac{\phi(\epsilon / 2)}{4 M}+\frac{\phi(\epsilon / 2)}{4 M}\right) M,
$$

so

$$
\phi\left(\left\|v_{n^{*}}-x^{*}\right\|\right)-2 \mu_{n^{*}}-\left(\alpha_{n^{*}}+2\left(k_{n^{*}}-1\right)\right) M \geq \phi\left(\frac{\epsilon}{2}\right)-\frac{3 \phi(\epsilon / 2)}{4}>0
$$

Moreover, $\phi\left(\left\|v_{n^{*}}-\mathrm{x}^{*}\right\|\right)>\phi(\epsilon / 2) / 2>0$, it results that

$$
\left\|v_{n^{*}+1}-x^{*}\right\|^{2} \leq \epsilon^{2}
$$

This is absurd. Thus, $\left\|v_{n^{*}+1}-x^{*}\right\|<\epsilon$.

In the same manner, by induction, one obtains that, for every $i \geq 1,\left\|v_{n^{*}+i}-x^{*}\right\|<\epsilon$. So $\left\|v_{n}-x^{*}\right\| \rightarrow 0$.

Remark 3.2. Our result is similar to Schu's theorem. However, our results hold in a more general setting of uniformly smooth Banach spaces, while the Schu's result holds for completely continuous, uniformly Lipschitzian mappings which are asymptotically pseudocontractive. 
Theorem 3.3. Let $X$ be a uniformly smooth Banach space, and let $T: X \rightarrow X$ be a continuous and asymptotically $\phi$-strongly pseudocontractive mapping with fixed point $x^{*}$ and bounded range.

Let $\left\{z_{n}\right\}$ and $\left\{z_{n}^{\prime}\right\}$ be the sequences defined by (1.14) and (1.15), respectively, where $\left\{\alpha_{n}\right\},\left\{\gamma_{n}\right\} \subset[0,1]$ are null sequences satisfying

(H1) $\lim _{n \rightarrow \infty} \alpha_{n}=0$ and $\gamma_{n}=o\left(\alpha_{n}\right)$,

(H2) $\sum_{n=1}^{\infty} \alpha_{n}=\infty$,

and such that $\alpha_{n} k_{n}<1$, for every $n \in \mathbb{N}$.

Let us suppose moreover that the sequences $\left\{w_{n}\right\},\left\{w_{n}^{\prime}\right\}$ are bounded in $X$.

Then, for any initial point $z_{0}^{\prime}, z_{0} \in X$, the following two assertions are equivalent.

(i) The Mann iteration sequence (1.14) converges to the fixed point $x^{*}$.

(ii) The modified implicit iteration sequence (1.15) converges to the fixed point $x^{*}$.

Proof. By the boundedness of the range of $T$ and by Lemma 2.4, one obtains that our schemes are bounded. Let us define

$$
M=\sup _{n}\left\{\begin{array}{c}
\left\|T^{n} z_{n}-T^{n} z_{n}^{\prime}\right\|,\left\|T^{n} z_{n}-z_{n}\right\|,\left\|T^{n} z_{n}^{\prime}-z_{n}^{\prime}\right\|,\left\|T^{n} z_{n}^{\prime}-z_{n-1}^{\prime}\right\| \\
\left\|z_{n+1}-z_{n}^{\prime}\right\|,\left\|w_{n}^{\prime}-z_{n-1}^{\prime}\right\|,\left\|w_{n}^{\prime}\right\|
\end{array}\right\} .
$$

By the iteration schemes (1.14) and (1.15), we have

$$
\begin{aligned}
\left\|z_{n+1}-z_{n}^{\prime}\right\|^{2} \leq & \left\|\left(1-\alpha_{n}\right)\left(z_{n}-z_{n-1}^{\prime}\right)+\alpha_{n}\left(T^{n} z_{n}-T^{n} z_{n}^{\prime}\right)+\gamma_{n}\left(z_{n-1}^{\prime}-w_{n}^{\prime}\right)\right\|^{2} \\
\leq & \left(1-\alpha_{n}\right)^{2}\left\|z_{n}-z_{n-1}^{\prime}\right\|^{2}+2\left\langle\alpha_{n}\left(T^{n} z_{n}-T^{n} z_{n}^{\prime}\right)+\gamma_{n}\left(z_{n-1}^{\prime}-w_{n}^{\prime}\right), j\left(z_{n+1}-z_{n}^{\prime}\right)\right\rangle \\
\leq & \left(1-\alpha_{n}\right)^{2}\left\|z_{n}-z_{n-1}^{\prime}\right\|^{2}+2 \alpha_{n}\left\langle T^{n} z_{n}-T^{n} z_{n}^{\prime}, j\left(z_{n}-z_{n}^{\prime}\right)\right\rangle \\
& +2 \alpha_{n}\left\langle T^{n} z_{n}-T^{n} z_{n}^{\prime}, j\left(z_{n+1}-z_{n}^{\prime}\right)-j\left(z_{n}-z_{n}^{\prime}\right)\right\rangle+2 \gamma_{n} M^{2} \\
\leq & \left(1-\alpha_{n}\right)^{2}\left\|z_{n}-z_{n-1}^{\prime}\right\|^{2}+2 \alpha_{n} k_{n}\left\|z_{n}-z_{n}^{\prime}\right\|^{2} \\
& -2 \alpha_{n} \phi\left(\left\|z_{n}-z_{n}^{\prime}\right\|\right)+2 \alpha_{n} M \sigma_{n}+2 \gamma_{n} M^{2},
\end{aligned}
$$

where $\sigma_{n}=\left\|j\left(z_{n+1}-z_{n}^{\prime}\right)-j\left(z_{n}-z_{n}^{\prime}\right)\right\|$. By (1.14), we have

$$
\left\|\left(z_{n+1}-z_{n}^{\prime}\right)-\left(z_{n}-z_{n}^{\prime}\right)\right\|=\left\|z_{n+1}-z_{n}\right\|=\left\|\alpha_{n}\left(T^{n} z_{n}-z_{n}\right)\right\| \leq \alpha_{n} M .
$$


It follows from (H1) that $\left\|\left(z_{n+1}-z_{n}^{\prime}\right)-\left(z_{n}-z_{n}^{\prime}\right)\right\| \rightarrow 0$ as $n \rightarrow \infty$, which implies that $\sigma_{n} \rightarrow 0$ as $n \rightarrow \infty$. Moreover, for all $n \geq 0$,

$$
\begin{aligned}
\left\|z_{n}-z_{n}^{\prime}\right\|^{2} & \leq\left(\left\|z_{n}-z_{n-1}^{\prime}\right\|+\left\|z_{n-1}^{\prime}-z_{n}^{\prime}\right\|\right)^{2} \\
& \leq\left(\left\|z_{n}-z_{n-1}^{\prime}\right\|+\alpha_{n}\left\|T^{n} z_{n}^{\prime}-z_{n-1}^{\prime}\right\|+\gamma_{n}\left\|w_{n}^{\prime}-z_{n-1}^{\prime}\right\|\right)^{2} \\
& \leq\left[\left\|z_{n}-z_{n-1}^{\prime}\right\|+\left(\alpha_{n}+\gamma_{n}\right) M\right]^{2} \\
& =\left\|z_{n}-z_{n-1}^{\prime}\right\|^{2}+\left(\alpha_{n}+\gamma_{n}\right)\left[2 M\left\|z_{n}-z_{n-1}^{\prime}\right\|+\left(\alpha_{n}+\gamma_{n}\right) M^{2}\right] \\
& \leq\left\|z_{n}-z_{n-1}^{\prime}\right\|^{2}+3\left(\alpha_{n}+\gamma_{n}\right) M^{2} .
\end{aligned}
$$

Again by the boundedness of all components, we have that

$$
\left\|z_{n}^{\prime}-z_{n-1}^{\prime}\right\|=\left\|\alpha_{n}\left(T^{n} z_{n}^{\prime}-z_{n-1}^{\prime}\right)+\gamma_{n}\left(w_{n}^{\prime}-z_{n-1}^{\prime}\right)\right\| \leq\left(\alpha_{n}+\gamma_{n}\right) M,
$$

and so

$$
\begin{aligned}
\left\|z_{n+1}-z_{n}^{\prime}\right\| & =\left\|\left(z_{n}-z_{n}^{\prime}\right)+\left(z_{n}^{\prime}-z_{n-1}^{\prime}\right)-\alpha_{n}\left(z_{n}-z_{n-1}^{\prime}\right)+\alpha_{n}\left(T^{n} z_{n}-T^{n} z_{n}^{\prime}\right)+\gamma_{n}\left(z_{n-1}^{\prime}-w_{n}^{\prime}\right)\right\| \\
& \leq\left\|z_{n}-z_{n}^{\prime}\right\|+\left\|z_{n}^{\prime}-z_{n-1}^{\prime}\right\|+\alpha_{n}\left\|z_{n}-z_{n-1}^{\prime}\right\|+\alpha_{n}\left\|T^{n} z_{n}-T^{n} z_{n}^{\prime}\right\|+\gamma_{n}\left\|z_{n-1}^{\prime}-w_{n}^{\prime}\right\| \\
& \leq\left\|z_{n}-z_{n}^{\prime}\right\|+3\left(\alpha_{n}+\gamma_{n}\right) M .
\end{aligned}
$$

Hence, we have that $\left\|z_{n}-z_{n}^{\prime}\right\| \geq\left\|z_{n+1}-z_{n}^{\prime}\right\|-e_{n}$, where $e_{n}=3\left(\alpha_{n}+\gamma_{n}\right) M$. Note that $e_{n} \rightarrow 0$ as $n \rightarrow \infty$. As in proof of Theorem 3.1, we distinguish two cases:

(i) the set of index for which $\left\|z_{n+1}-z_{n}^{\prime}\right\|-e_{n} \leq 0$ contains infinite terms,

(ii) the set of index for which $\left\|z_{n+1}-z_{n}^{\prime}\right\|-e_{n} \leq 0$ contains finite terms.

In the first case (i), we can extract a subsequence such that $\left\|z_{n_{k}}-z_{n_{k}-1}^{\prime}\right\| \rightarrow 0$, as $k \rightarrow \infty$. Substituting (3.20) in (3.18), we have that

$$
\begin{aligned}
\left\|z_{n+1}-z_{n}^{\prime}\right\|^{2} \leq & \left(1+\alpha_{n}^{2}-2 \alpha_{n}\right)\left\|z_{n}-z_{n-1}^{\prime}\right\|^{2}+2 \alpha_{n} k_{n}\left\|z_{n}-z_{n-1}^{\prime}\right\|^{2}+6\left(\alpha_{n}+\gamma_{n}\right) M^{2} \alpha_{n} k_{n} \\
& -2 \alpha_{n} \phi\left(\left\|z_{n}-z_{n}^{\prime}\right\|\right)+2 \alpha_{n} M \sigma_{n}+2 \gamma_{n} M^{2} \\
\leq & \left\|z_{n}-z_{n-1}^{\prime}\right\|^{2}+\left(\alpha_{n}^{2}+2 \alpha_{n}\left(k_{n}-1\right)\right)\left\|z_{n}-z_{n-1}^{\prime}\right\|^{2}+6\left(\alpha_{n}+\gamma_{n}\right) M^{2} \alpha_{n} \bar{k} \\
& -2 \alpha_{n} \phi\left(\left\|z_{n}-z_{n}^{\prime}\right\|\right)+2 \alpha_{n} M \sigma_{n}+2 \gamma_{n} M^{2} \\
\leq & \left\|z_{n}-z_{n-1}^{\prime}\right\|^{2}-\alpha_{n} \phi\left(\left\|z_{n}-z_{n}^{\prime}\right\|\right)+2 \gamma_{n} M^{2} \\
& -\alpha_{n}\left[\phi\left(\left\|z_{n}-z_{n}^{\prime}\right\|\right)-\alpha_{n} M^{2}-2 M^{2}\left(k_{n}-1\right)-6\left(\alpha_{n}+\gamma_{n}\right) M^{2} \bar{k}-2 M \sigma_{n}\right] \\
= & \left\|z_{n}-z_{n-1}^{\prime}\right\|^{2}-\alpha_{n} \phi\left(\left\|z_{n}-z_{n}^{\prime}\right\|\right)+2 \gamma_{n} M^{2} \\
& -\alpha_{n}\left[\phi\left(\left\|z_{n}-z_{n}^{\prime}\right\|\right)-7 \bar{k} \alpha_{n} M^{2}-2 M^{2}\left(k_{n}-1\right)-6 \gamma_{n} M^{2} \bar{k}-2 M \sigma_{n}\right],
\end{aligned}
$$


where $\bar{k}=\sup _{n} k_{n}$. Again by (3.20), for every $\epsilon>0$, there exists an index $l$ such that, if $j>l$,

$$
\left\|z_{n_{j}}-z_{n_{j}-1}^{\prime}\right\|<\epsilon, \quad\left\|z_{n_{j}}-z_{n_{j}}^{\prime}\right\|<2 \epsilon
$$

By hypotheses on the control sequence, with the same $\epsilon>0$, there exists an index $N$ such that definitively

$$
\begin{gathered}
\left|\alpha_{n}\right|<\min \left\{\frac{\epsilon}{12 M}, \frac{\phi(\epsilon / 2)}{56 M^{2} \bar{k}}\right\} \\
\left|\gamma_{n}\right|<\min \left\{\frac{\epsilon}{12 M}, \frac{\phi(\epsilon / 2)}{48 M^{2} \bar{k}}\right\}, \quad\left|\frac{\gamma_{n}}{\alpha_{n}}\right|<\frac{\phi(\epsilon / 2)}{4 M^{2}}, \\
\left|k_{n}-1\right|<\frac{\phi(\epsilon / 2)}{16 M^{2}}, \\
\left|\sigma_{n}\right|<\frac{\phi(\epsilon / 2)}{16 M} .
\end{gathered}
$$

So take $n^{*}>\max \left\{n_{l}, N\right\}$ with $n^{*}=n_{j}$ for a certain $j$.

We can prove that $\left\|z_{n+1}-z_{n}^{\prime}\right\| \rightarrow 0$ as $n \rightarrow \infty$ proving that, for every $i \geq 0$ it results $\left\|z_{n^{*}+i}-z_{n^{*}+i-1}^{\prime}\right\|<\epsilon$.

Let $i=1$. If we suppose that $\left\|z_{n^{*}+1}-z_{n^{*}}^{\prime}\right\| \geq \epsilon$, it results that

$$
\epsilon \leq\left\|z_{n^{*}+1}-z_{n^{*}}^{\prime}\right\| \leq\left\|z_{n^{*}}-z_{n^{*}}^{\prime}\right\|+3\left(\alpha_{n^{*}}+\gamma_{n^{*}}\right) M<\left\|z_{n^{*}}-z_{n^{*}}^{\prime}\right\|+\frac{\epsilon}{2}
$$

so $\left\|z_{n^{*}}-z_{n^{*}}^{\prime}\right\|>\epsilon / 2$. In consequence of this, $\phi\left(\left\|z_{n^{*}}-z_{n^{*}}^{\prime}\right\|\right)>\phi(\epsilon / 2)$.

In (3.23), we note that

$$
\begin{aligned}
7 \bar{k} \alpha_{n^{*}} & M^{2}+2 M^{2}\left(k_{n^{*}}-1\right)+6 \gamma_{n^{*}} M^{2} \bar{k}+2 M \sigma_{n^{*}} \\
& \leq 7 \bar{k} M^{2} \frac{\phi(\epsilon / 2)}{56 M^{2} \bar{k}}+2 M^{2} \frac{\phi(\epsilon / 2)}{16 M^{2}}+6 M^{2} \bar{k} \frac{\phi(\epsilon / 2)}{48 M^{2} \bar{k}}+2 M \frac{\phi(\epsilon / 2)}{16 M} \\
& =\frac{\phi(\epsilon / 2)}{8} 4=\frac{\phi(\epsilon / 2)}{2},
\end{aligned}
$$

so

$$
\phi\left(\left\|z_{n^{*}}-z_{n^{*}}^{\prime}\right\|\right)-7 \bar{k} \alpha_{n^{*}} M^{2}-2 M^{2}\left(k_{n^{*}}-1\right)-6 \gamma_{n^{*}} M^{2} \bar{k}-2 M \sigma_{n^{*}} \geq \frac{\phi(\epsilon / 2)}{2}
$$

Hence, in (3.23), remains

$$
\left\|z_{n^{*}+1}-z_{n^{*}}^{\prime}\right\|^{2} \leq \epsilon^{2}-\alpha_{n^{*}} \phi\left(\left\|z_{n^{*}}-z_{n^{*}}^{\prime}\right\|\right)+2 \gamma_{n^{*}} M^{2} .
$$


In the same manner,

$$
\phi\left(\left\|z_{n^{*}}-z_{n^{*}}^{\prime}\right\|\right)-2 \frac{\gamma_{n^{*}}}{\alpha_{n^{*}}} M^{2}>\phi\left(\frac{\epsilon}{2}\right)-\frac{\phi(\epsilon / 2)}{2}>0
$$

so

$$
\left\|z_{n^{*}+1}-z_{n^{*}}^{\prime}\right\|^{2} \leq \epsilon^{2}
$$

This is absurd. By the same idea and by using the induction method, we obtain that $\left\|z_{n^{*}+i}-z_{n^{*}+i-1}^{\prime}\right\|<\epsilon$, for every $i \geq 0$. This assure that $\left\|z_{n+1}-z_{n}^{\prime}\right\| \rightarrow 0$. In the second case (ii), definitively, $\left\|z_{n+1}-z_{n}^{\prime}\right\|-e_{n} \geq 0$, then, from the strict increasing function $\phi$, we have

$$
\phi\left(\left\|z_{n}-z_{n}^{\prime}\right\|\right) \geq \phi\left(\left\|z_{n+1}-z_{n}^{\prime}\right\|-e_{n}\right) .
$$

Substituting (3.32) and (3.20) into (3.18) and simplifying, we have

$$
\begin{aligned}
\left\|z_{n+1}-z_{n}^{\prime}\right\|^{2} \leq & \left\|z_{n}-z_{n-1}^{\prime}\right\|^{2}+\alpha_{n}^{2}\left\|z_{n}-z_{n-1}^{\prime}\right\|^{2}+2 \alpha_{n}\left(k_{n}-1\right)\left\|z_{n}-z_{n-1}^{\prime}\right\|^{2} \\
& +6 \alpha_{n} k_{n}\left(\alpha_{n}+\gamma_{n}\right) M^{2}-2 \alpha_{n} \phi\left(\left\|z_{n+1}-z_{n}^{\prime}\right\|-e_{n}\right)+2 \alpha_{n} \sigma_{n} M+2 \gamma_{n} M^{2} \\
\leq & \left\|z_{n}-z_{n-1}^{\prime}\right\|^{2}-2 \alpha_{n} \phi\left(\left\|z_{n+1}-z_{n}^{\prime}\right\|-e_{n}\right)+\alpha_{n}^{2} M^{2} \\
& +2 \alpha_{n}\left(k_{n}-1\right) M^{2}+6 \alpha_{n} k_{n}\left(\alpha_{n}+\gamma_{n}\right) M^{2}+2 \alpha_{n} \sigma_{n} M+2 \gamma_{n} M^{2} .
\end{aligned}
$$

By virtue of Lemma 2.3, we obtain that $\lim _{n \rightarrow \infty}\left\|z_{n}-z_{n-1}^{\prime}\right\|=0$.

\section{References}

[1] K. Goebel and W. A. Kirk, "A fixed point theorem for asymptotically nonexpansive mappings," Proceedings of the American Mathematical Society, vol. 35, pp. 171-174, 1972.

[2] F. E. Browder, "Nonexpansive nonlinear operators in a Banach space," Proceedings of the National Academy of Sciences of the United States of America, vol. 54, pp. 1041-1044, 1965.

[3] J. Schu, "Iterative construction of fixed points of asymptotically nonexpansive mappings," Journal of Mathematical Analysis and Applications, vol. 158, no. 2, pp. 407-413, 1991.

[4] E. U. Ofoedu, "Strong convergence theorem for uniformly L-Lipschitzian asymptotically pseudocontractive mapping in real Banach space," Journal of Mathematical Analysis and Applications, vol. 321, no. 2, pp. 722-728, 2006.

[5] H. Zhou, "Demiclosedness principle with applications for asymptotically pseudo-contractions in Hilbert spaces," Nonlinear Analysis. Theory, Methods E Applications, vol. 70, no. 9, pp. 3140-3145, 2009.

[6] L.-C. Ceng, Q. H. Ansari, and J.-C. Yao, "Strong and weak convergence theorems for asymptotically strict pseudocontractive mappings in intermediate sense," Journal of Nonlinear and Convex Analysis. An International Journal, vol. 11, no. 2, pp. 283-308, 2010.

[7] L.-C. Ceng, A. Petruşel, and J.-C. Yao, "Iterative approximation of fixed points for asymptotically strict pseudocontractive type mappings in the intermediate sense," Taiwanese Journal of Mathematics, vol. 15, no. 2, pp. 587-606, 2011.

[8] L. C. Ceng, A. Petrusel, and J. C. Yao, "A hybrid method for Lipschitz continuous monotone mappings and asymptotically strict pseudocontractive mappings in the intermediate sense," Journal of Nonlinear and Convex Analysis, vol. 11, no. 1, 2010.

[9] C. Chidume, Geometric Properties of Banach Spaces and Nonlinear Iterations, Springer, London, UK, 2009. 
[10] D. R. Sahu, H.-K. Xu, and J.-C. Yao, “Asymptotically strict pseudocontractive mappings in the intermediate sense," Nonlinear Analysis. Theory, Methods E Applications, vol. 70, no. 10, pp. 3502-3511, 2009.

[11] Z.-h. Sun, "Strong convergence of an implicit iteration process for a finite family of asymptotically quasi-nonexpansive mappings," Journal of Mathematical Analysis and Applications, vol. 286, no. 1, pp. 351-358, 2003.

[12] K. Deimling, "Zeros of accretive operators," Manuscripta Mathematica, vol. 13, pp. 365-374, 1974.

[13] M. O. Osilike, "Iterative solution of nonlinear equations of the $\phi$-strongly accretive type," Journal of Mathematical Analysis and Applications, vol. 200, no. 2, pp. 259-271, 1996.

[14] K. Deimling, Nonlinear Functional Analysis, Springer, Berlin, Germany, 1985.

[15] L.S. Liu, "Ishikawa and Mann iterative process with errors for nonlinear strongly accretive mappings in Banach spaces," Journal of Mathematical Analysis and Applications, vol. 194, no. 1, pp. 114-125, 1995.

[16] S. S. Chang, "Some results for asymptotically pseudo-contractive mappings and asymptotically nonexpansive mappings," Proceedings of the American Mathematical Society, vol. 129, no. 3, pp. 845-853, 2001.

[17] S. S. Chang, K. K. Tan, H. W. J. Lee, and C. K. Chan, "On the convergence of implicit iteration process with error for a finite family of asymptotically nonexpansive mappings," Journal of Mathematical Analysis and Applications, vol. 313, no. 1, pp. 273-283, 2006.

[18] F. Gu, "The new composite implicit iterative process with errors for common fixed points of a finite family of strictly pseudocontractive mappings," Journal of Mathematical Analysis and Applications, vol. 329, no. 2, pp. 766-776, 2007.

[19] Z. Huang, "Equivalence theorems of the convergence between Ishikawa and Mann iterations with errors for generalized strongly successively $\phi$-pseudocontractive mappings without Lipschitzian assumptions," Journal of Mathematical Analysis and Applications, vol. 329, no. 2, pp. 935-947, 2007.

[20] Z. Huang and F. Bu, "The equivalence between the convergence of Ishikawa and Mann iterations with errors for strongly successively pseudocontractive mappings without Lipschitzian assumption," Journal of Mathematical Analysis and Applications, vol. 325, no. 1, pp. 586-594, 2007.

[21] C. E. Chidume and M. O. Osilike, "Equilibrium points for a system involving $m$-accretive operators," Proceedings of the Edinburgh Mathematical Society, vol. 44, no. 1, pp. 187-199, 2001.

[22] C. E. Chidume and H. Zegeye, "Approximate fixed point sequences and convergence theorems for asymptotically pseudocontractive mappings," Journal of Mathematical Analysis and Applications, vol. 278, no. 2, pp. 354-366, 2003.

[23] Y. Xu, "Ishikawa and Mann iterative processes with errors for nonlinear strongly accretive operator equations," Journal of Mathematical Analysis and Applications, vol. 224, no. 1, pp. 91-101, 1998.

[24] P.-E. Maingé, "Approximation methods for common fixed points of nonexpansive mappings in Hilbert spaces," Journal of Mathematical Analysis and Applications, vol. 325, no. 1, pp. 469-479, 2007.

[25] C. E. Chidume and C. O. Chidume, "Convergence theorem for zeros of generalized lipschitz generalized $\phi$-quasi-accretive operators," Proceedings of the American Mathematical Society, vol. 134, no. 1, pp. 243-251, 2006. 


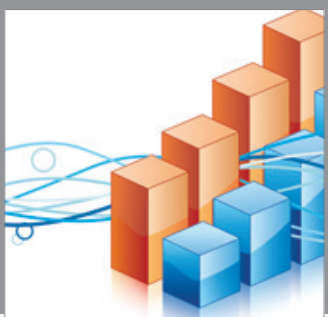

Advances in

Operations Research

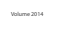

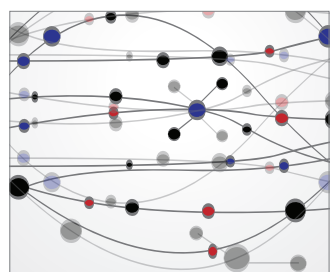

\section{The Scientific} World Journal
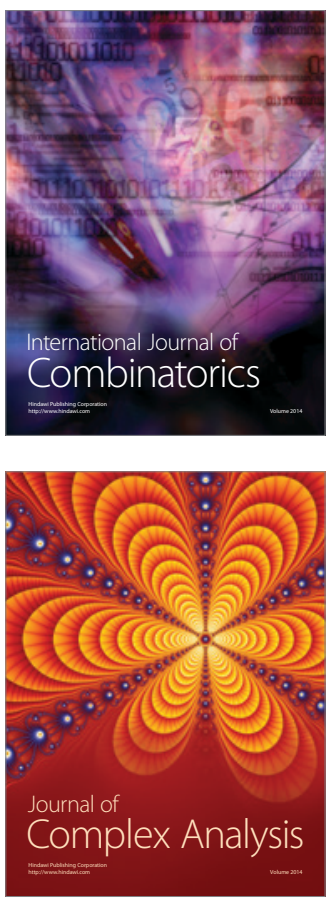

International Journal of

Mathematics and

Mathematical

Sciences
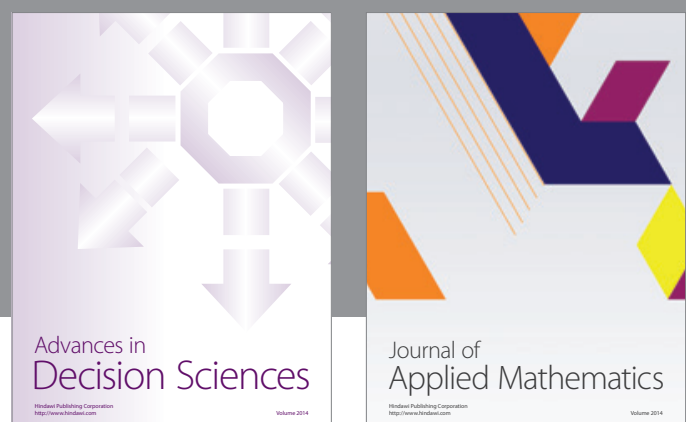

Journal of

Applied Mathematics
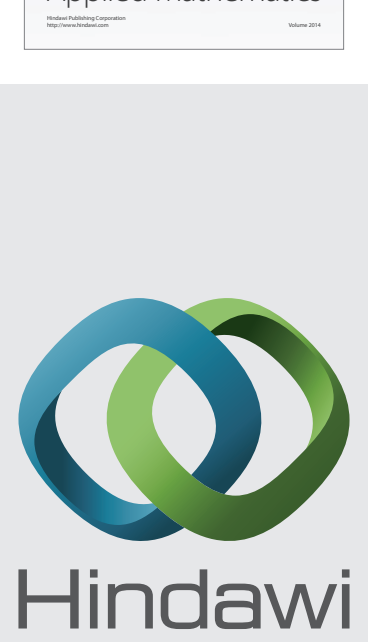

Submit your manuscripts at http://www.hindawi.com
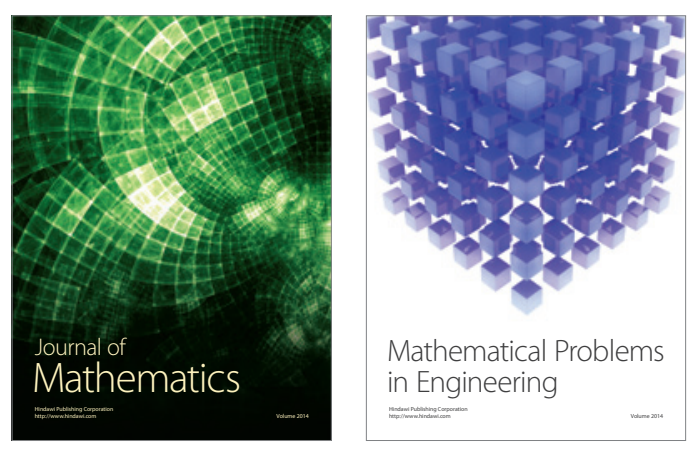

Mathematical Problems in Engineering
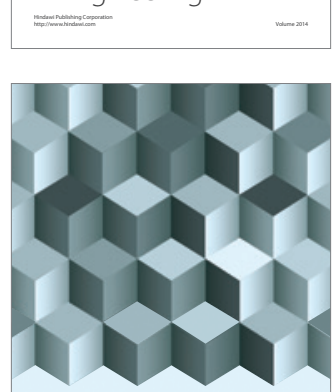

Journal of

Function Spaces
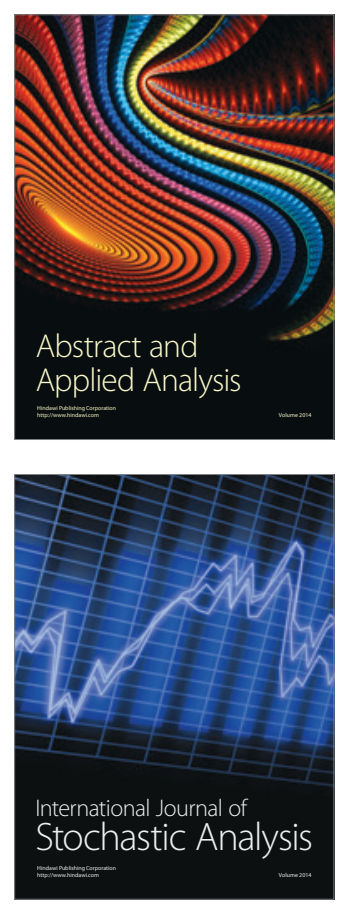

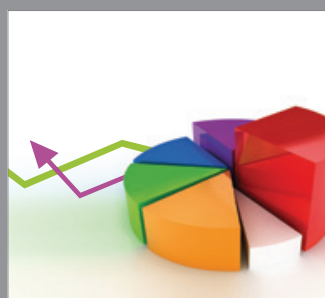

ournal of

Probability and Statistics

Promensencen
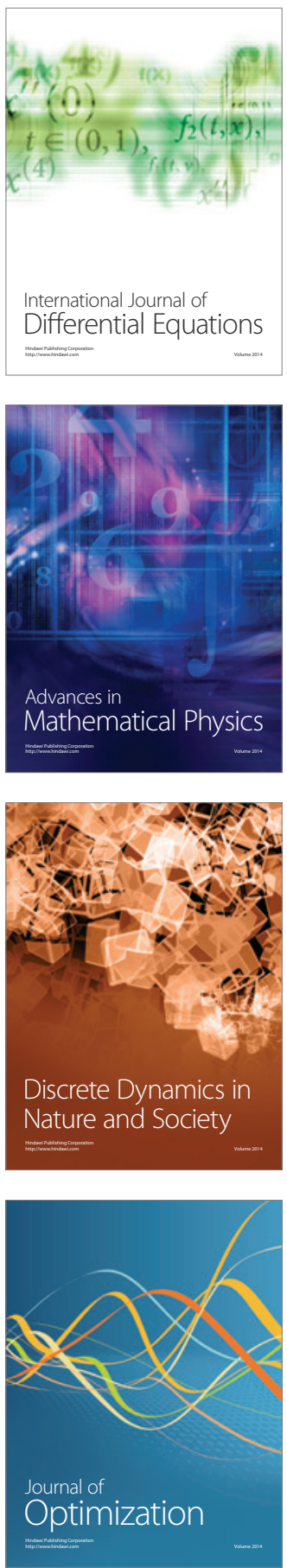